\title{
Order in the midst of chaos
}

Complex systems, as different as neural networks and clusters of interacting galaxies, are susceptible to chaotic behaviour. Numerical calculations now suggest that some degree of order may persist beneath the chaos.

WHY is the study of complex systems offered as a means of solving all the outstanding problems of the real world? The explanation is not all that deeply hidden. The complex problems of the real world may have more in common with each other than with any of the simple problems dealt with elegantly in the textbooks. A neural network, for example, is a large number of neurons of which all interact with all others, so that people are reduced either to making generalizations about their expected behaviour or to making numerical calculations of particular systems. Globular clusters, or clusters of interacting galaxies, are in much the same case, as are the problems of fluid dynamics in the real world. All these systems, for example, share a propensity to run to chaos.

This is the spirit in which Kunihiko Kaneko from the University of Tokyo (and, in particular, from the teachingorientated College of Arts and Sciences in which undergraduates spend their first two years) has managed rigorously to demonstrate a general property of systems of this kind (Phys. Rev. Lett. 65, 1391; 1990). He says that neural networks and fluid dynamics are among the problems to which his calculations are relevant. But his result is interesting and important - that order can persist even when a complex system has lapsed into chaos.

Those wishing to follow Kaneko's argument had better start with a paper published a year ago (Phys. Rev. Lett. 63, 219; 1989) which, among other things, contains both the notation for handling the problem and the inspiration for the study now described. The most arresting of his conclusions, then, was a kind of phase diagram outlining the relationship between chaotic states of a complex system with global coupling in which the phases are characterized by differing degrees of persistent order.

Formally, there is no great difficulty in setting up the problem. If there are $N$ elements in a complex system, the time evolution of a property $x_{j}$ of the $j$ th element will be determined primarily by that element's autonomous evolution, but also by its interactions with all the other elements in the system. So, if time is measured as a multiple $n$ of some unit interval, the problem is simply that of relating $x_{i}(n+1)$ to the values of all the $x_{k}(n)$ s one time-step earlier. Formally again, $\quad x_{i}(n+1)$ is predominantly $(1-\varepsilon) f\left(x_{j}(n)\right)$ on account of its autonomous evolution, and a sum of terms such as $(\varepsilon / N) f\left(x_{k}(n)\right)$ on account of the interaction, where $f(x)$ is some function and $\varepsilon$ is a number small compared with 1 .

The fun begins with the choice of the function $f$, which, if linear, yields nothing remarkable, but which is well known to yield chaotic behaviour if taken to be, say, $f(x)=1-a x^{2}$ (where $a$ is again a constant) with the rule that the integral part of any calculated $x$ is discarded. The outcome, now one of many textbook examples of how to generate chaos, has the effect of mapping the range of numbers between -1 and +1 onto itself with each successive iteration, and also has the convenient property that one can regard positive and negative values of the variable $x_{i}$ as simply minuses and pluses, corresponding to, say, OFF and ON states of a neuron, according to whether they lie below or above a characteristic value of the parameter $a$.

Kaneko's results are not dependent on this choice of function; he has been able to show that the same general pattern of behaviour crops up with other functions. But the dynamical rule specifying the evolution of the system is rather special, in that all the interactions between different elements have the same form and the same strength. Those assumptions evidently oversimplify the properties of, say, a true neural network or globular cluster, but there is no reason to suppose that the assumption is critical to what emerges.

And what is that? The conclusion that order is curiously immune to the onset of chaos. What Kaneko showed last year is that coherent oscillation of a system from PLUS to MINUS is surprisingly common, but that appropriate values of the parameters $\varepsilon$ and $a$ cause it to break up into two clusters (defined conceptually, not geometrically) which oscillate out of phase with each other, members of one set going from PLUS to MINUS while the others go from MINUS to pLus. These persistent states of chaotic oscillation are the 'phases' provided by the model. What seems to be happening is that, for each element, there is a competition between its tendency to chaotic instability and its tendency to conformity arising from the averaging effect of the system of elements as a whole. The greater the nonlinearity (parameter $a$ ), the more widespread the disorder; the greater the averaging effect (parameter $\varepsilon$ ), the more persistent is

\section{coherence}

Kaneko's article last year raises a host of questions that have not yet been answered, but his new calculation answers one of them in a fashion that raises further intriguing questions. However remote the model may seem from the models of classical dynamics, it does at least provide an easy way of going over to a mean-field representation of the system in the Landau and Ginzburg sense. Indeed, the quantity $(1 / N) \Sigma_{j} f\left(x_{j}(n)\right)$ represents a kind of ordering mean field whose statistical properties can be calculated numerically. What emerges is that, for each set of parameters, the statistical distribution of the mean field is an inverted bell-shape centred on some numerical value and that there is a lower limit to the width of the inverted bell (or to the variance of the mean field) with increasing complexity (the value of $N$ ). That is what would be expected from the central limit theorem of statistics.

The surprise is what happens to the mean-square deviation of the mean field, which would naively be expected to fall to zero with increasing complexity by what is still quaintly called the law of large numbers. The numerical experiments show that, for some values of the parameters, the mean-square deviation of the mean field falls off not to zero, but to some constant value which is, of necessity, greater than zero. What can be the explanation? Order in the midst of chaos. Kaneko shows this to be the case by calculating a simple statistic that measures the mutual correlation of the states of elements in his model of the complex system. For good measure, he shows that the expectations of the law of large numbers are fulfilled if a little noise is added to the system, but that reality does not approach expectation as $1 / N$, but as some power of that factor less than one.

That chaotic behaviour does not necessarily imply that everything is random is borne out by the well-known regularities in the way in which chaos develops - for example, by bifurcation of period-doubling, for all the world as if the development of chaos is an orderly process. The recognition that the chaotic states of a system may embody a measure of persistent coherence may be even more significant if, say, it is a way of accounting for persistence of neural memory in disordered neural networks.

John Maddox 